\title{
PI3K/mTOR Kinase Inhibitor PF-04691502
}

National Cancer Institute

\section{Source}

National Cancer Institute. PI3K/mT OR Kinase Inhibitor PF-04691502. NCI Thesaurus.

Code C84837.

An agent targeting the phosphatidylinositol 3 kinase (PI3K) and mammalian targ et of rapamycin (mTOR) in the PI3K/mT OR signaling pathway, with potential antineoplastic activity. PI3K/mT OR kinase inhibitor PF-04691502 inhibits both PI3K and mT OR kinases, which may result in apoptosis and growth inhibition of cancer cells overexpressing $\mathrm{PI3K} / \mathrm{mT}$ OR. Activation of the PI3K/mT OR pathway promotes cell growth, survival, and resistance to chemotherapy and radiotherapy; mTOR, a serine/threonine kinase downstream of PI3K, may also be activated independent of PI3K. 